\title{
Torsades de Pointes Complicating Pentamidine Therapy of Pneumocystis Carinii Pneumonia in Acute Myelogenous Leukemia
}

\author{
Mami Otsuka, Heiwa Kanamori, Shin Sasaki, Jun Taguchi, Hiroshi Harano, Kouji Ogawa, \\ Michio Matsuzaki*, Hiroshi Mohri, Takao Okubo, Shinichi Sumita** and Hisao Ochiai**
}

Pentamidine isethionate induced torsades de pointes in a 33-year-old woman with acute myelogenous leukemia. This is the first report of Pentamidine-induced torsades de pointes in Japan for over ten years. On the 4th day of intravenous pentamidine for Pneumocystis carinii pneumonia, asymptomatic sinus bradycardia was noted with QT interval prolongation, and torsades de pointes were revealed on the 8th day. Although torsades de pointes was dissolved with discontinuation of the intravenous pentamidine and administration of magnesium sulfate, sinus bradycardia and prolonged QT interval persisted. Ventricular pacing resulted in no arrhythmia and normalization of the $Q T$ interval on the 10th day after discontinuation of pentamidine. Careful monitoring of the electrocardiogram should be carried out during intravenous pentamidine therapy.

(Internal Medicine 36: 705-708, 1997)

Key words: bradycardia, prolonged QT interval, cardiac pacing

\section{Introduction}

Although pentamidine isethionate is widely used for treatment of Pneumocystis carinii pneumonia (PCP) in patients with acquired immunodeficiency syndrome (AIDS), a high incidence of adverse reactions of intravenous pentamidine therapy has been reported (1-4). The most life-threatening side effect is cardiotoxicity (3), which may be manifested by sinus bradycardia, prolongation of the QT interval and ventricular arrhythmia including torsades de pointes.

We describe a case of torsades de pointes caused by intravenous pentamidine therapy for PCP in a patient with acute myelogenous leukemia (AML).

\section{Case Report}

A 33-year-old female was admitted for treatment of AML in April 1995. Complete remission had been achieved by an induction chemotherapy (behenoylara-C, daunorubicin, 6mercaptopurine, and prednisolone). She had no history or physical findings indicative of heart disease. The electrocardiogram (ECG) on admission showed sinus rhythm with heart rate of 72/min and without prolongation of the QT interval (QTc $=415$ $\mathrm{msec}$ ) (Fig. 1). Serum potassium and magnesium were normal. On the 21st day following the second consolidation therapy with cytosine arabinoside and mitoxantron, high fever appeared despite administration of antibiotics and anti-mold agents and the white blood cell count was $200 / \mu 1$. Ten days later, she became dyspneic with severe hypoxemia $\left(\mathrm{PaO}_{2} 35.4\right.$ torr) by arterial blood gas analysis. A chest roentgenogram showed bilateral fluffy infiltrates, indicating interstitial pneumonia. Methylprednisolone-pulse therapy, recombinant granulocyte-colony stimulating factor and immunoglobulin were administrated. In addition, pentamidine isethionate, $4 \mathrm{mg}$ / $\mathrm{kg}$ per day intravenously, was used. Her symptoms and pulmonary interstitial infiltrates were improved within a few days. On the 4th day after pentamidine therapy, ECG showed bradycardia $(40-50 / \mathrm{min})$ with QT interval prolongation $(\mathrm{QTc}=470$ $\mathrm{msec}$ ). Then sinus bradycardia progressed up to $38 / \mathrm{min}$ with occasional ventricular ectopic beats accompanied with prolonged QTc interval, flattened to biphasic $T$ waves and $U$ wave fusing with preceding $\mathrm{T}$ wave in ECG (Fig. 2). On the next day, she had episodes of torsades de pointes with chest discomfort (Fig. 3). The serum potassium, calcium, and magnesium were

From the First Department of Internal Medicine, Yokohama City University School of Medicine, Yokohama, *the Division of Blood Transfusion, Yokohama City University Hospital, Yokohama and **the Second Department of Internal Medicine, Yokohama City University School of Medicine, Yokohama Received for publication December 10, 1996; Accepted for publication May 30, 1997

Reprint requests should be addressed to Dr. Mami Otsuka, the First Department of Internal Medicine, Yokohama City University School of Medicine, 3-9 Fukuura, Kanazawa-ku, Yokohama 236 
I

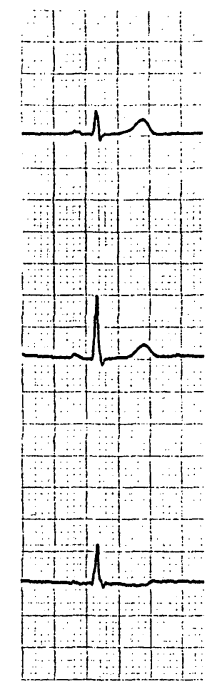

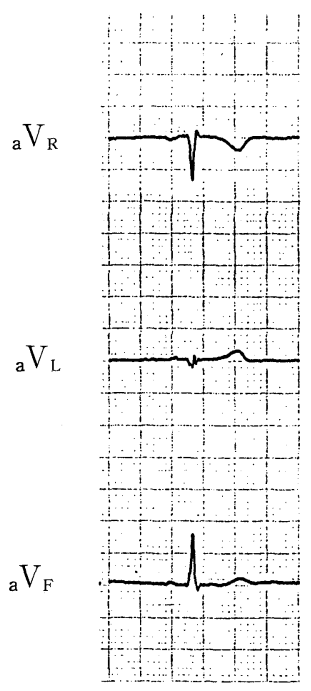
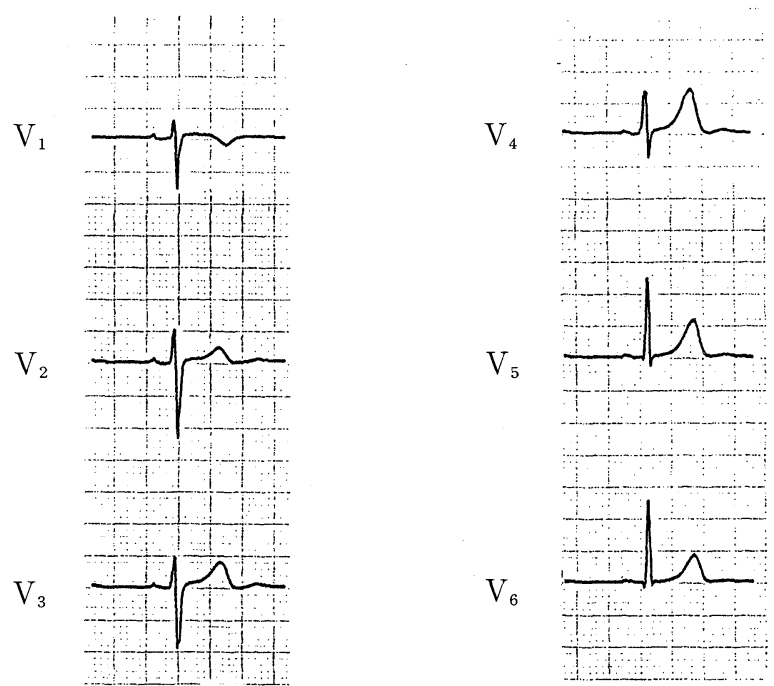

Figure 1. Electrocardiogram on admission.
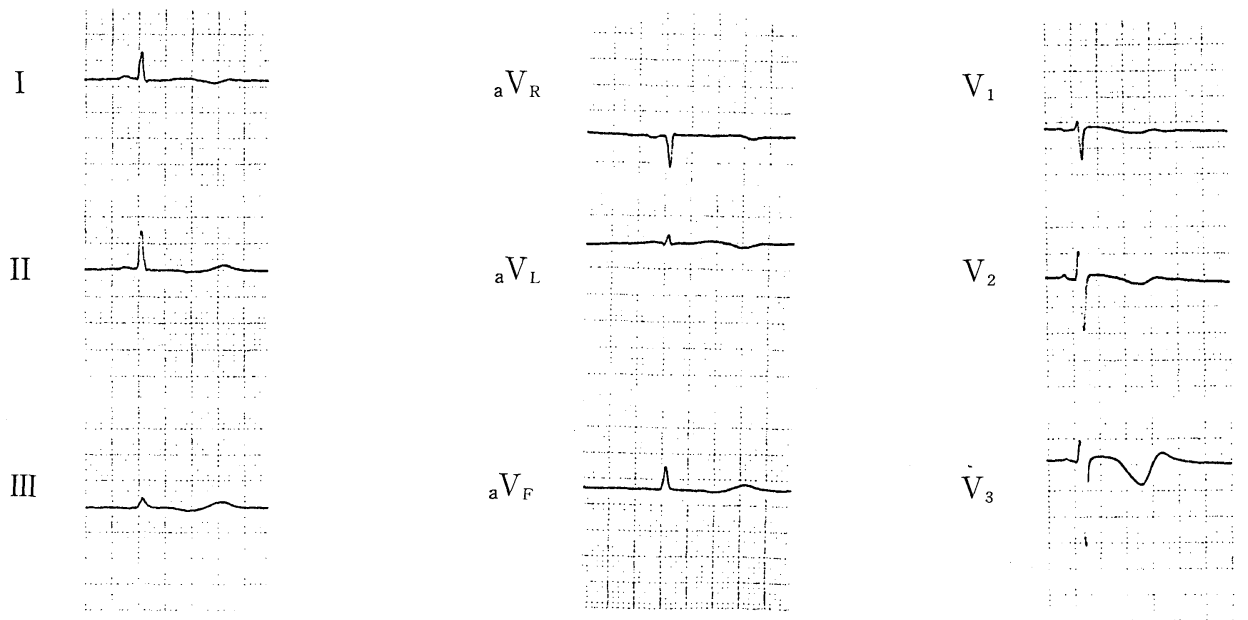

$\mathrm{V}_{4}$

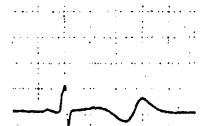

$\mathrm{V}_{5}$

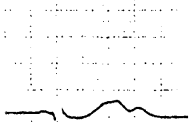

Figure 2. Prolonged QT interval and biphasic $T$ wave were detected on the 4th day after intravenous pentamidine therapy.

II

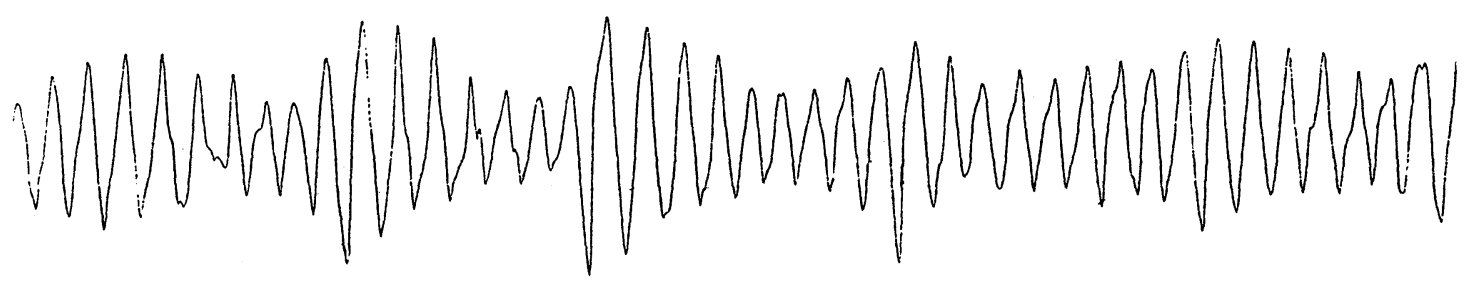

Figure 3. Torsades de pointes were seen on the 8th day after pentamidine therapy.

$3.4 \mathrm{mEq} / l, 8.8 \mathrm{mg} / \mathrm{dl}$ and, $1.8 \mathrm{mg} / \mathrm{dl}$, respectively. Magnesium sulfate and pentamidine in aerosol form for PCP in place of intravenous therapy were administrated. Although torsades de pointes was disappeared following the treatment, sinus brady- cardia with prolongation of the QT interval was persistent. She received ventricular pacing with a temporally pacemaker. Discontinuation of intravenous pentamidine for ten days normalized the QTc interval at $398 \mathrm{msec}$. No arrhythmia was seen after 
removal of temporally pacemaker. The patient had no recurrence of arrhythmia during follow-up of 11 months.

\section{Discussion}

In recent reviews (5-7), there have been reports of serious arrhythmia as increasing cases of PCP in patients with AIDS have resulted in a dramatic increase in the use of pentamidine since 1981. Pentamidine-induced arrhythmia has been rarely described in patients other than those with AIDS. For our knowledge, this is the first report of torsades de pointes caused by intravenous pentamidine therapy for PCP in a patient with AML. We believe that the main cause of the cardiotoxicity might have been the pentamidine in this case, although the interaction with other drugs including methylprednisolone, granylocyte colony-stimulating factor, and immunoglobulin is unclear.

Various complications including impaired glucose metabolism, acute pancreatitis, hepatic dysfunction, neuropathy, nephrotoxicity and hypotension have been shown in patients receiving pentamidine $(8,9)$. The present patient did not have any adverse reactions except for the episode of arrhythmia. The spectrum of reported cardiotoxicity associated with pentamidine has been manifested by sinus bradycardia, prolonged QT interval, ventricular bigeminy, ventricular tachycardia and polymorphic ventricular tachycardia (torsades de pointes) (1-9). The proarrhythmic effects of pentamidine have been postulated to relate to its structural similarity with procainamide (3). Furthermore, electrolyte abnormalities and malnutrition may contribute to the pathogenesis of cardiotoxicity in patients with AIDS and PCP $(10,11)$, as may human immunodeficiency virus-mediated myocarditis (12). Although the genesis of cardiotoxicity by pentamidine is not clearly understood, prolongation of the QT interval is thought to be a common precursor of torsades de pointes $(3,5,13-15)$. The prolongation of QT interval and sinus bradycardia occurred on the 4th day after administration of pentamidine, and torsades de pointes developed on the 8th day in the present case. The cumulative dose of $28 \mathrm{mg} / \mathrm{kg}$ in the patient was lower than that of $56 \mathrm{mg} /$ $\mathrm{kg}$ recommended by the Center for Disease Control (16). Gonzales et al have demonstrated that there is no significant dose-dependent effect of pentamidine on the ECG in prospective studies in patients with AIDS and PCP (4). Eisenhayer et al have described that pentamidine-associated QTc prolongation and torsades de pointes appear to be idiosyncratic phenomena rather than cumulative dose-related effects in their prospective study (15). Since pentamidine is avidly bound to tissue (17), prolonged therapy presumably contributes to this adverse reaction. According to a review of reported cases by Mani et al (6), the average duration of intravenous pentamidine before the appearance of torsades de pointes was 12.5 days with a range of 6-20 days. The relatively delayed onset and longer duration of arrhythmia after discontinuation are specific features in the setting of cardiotoxicity linked with pentamidine. Inf act, the prolonged QT interval persisted 10 days after disruption of intravenous administration in our patient.
It is well documented that certain electrolyte abnormalities contribute to the onset of pentamidine-induced torsades de pointes during pentamidine treatment of PCP $(2-4,10,11)$. However, the arrhythmia has also been reported in patients without electrolyte abnormalities $(14,15,18)$. In our case, mild hypomagnesemia was detected and thereby may increase the potential for proarrhythmia in the setting of receiving pentamidine. Administration of magnesium sulfate and discontinuation of intravenous pentamidine therapy were effective to improve torsades de pointes, but sinus bradycardia and the QT interval prolongation persisted. Tzivoni et al have reported the efficiency of magnesium sulfate for torsades de pointes with a prolonged QT interval (19). They also described that no significant changes were observed in the QT interval despite preventing the recurrence of torsades de pointes. Isoproterenol, atropine or cardiac pacing are used to shorten the delayed repolarization by increasing the heart rate in the treatment of torsades de pointes.

The patient received ventricular pacing with a temporally pacemaker for persistence of sinus bradycardia and QT interval prolongation. It has been well established that cardiac pacing is an effective and safe procedure in the treatment of torsades de pointes $(20,21)$. Cardiac pacing seems to be an useful application for preventing the recurrence of arrhythmia in view of prolonged cardiotoxicity after discontinuation of pentamidine.

Taylor et al have reported the safe and successful completion of therapy with pentamidine in aerosol form in a patient in whom torsades de pointes developed from the administration of intravenous pentamidine (22). In the present case, the aerosol form of pentamidine was administrated for two weeks (alternate days) in place of intravenous therapy and there was no recurrence of PCP.

As the use of pentamidine therapy for PCP in immunocompromized patients increases, we should be aware of the potential cardiac toxicity of pentamidine even within the recommended dose range. We believe that continual ECG should be monitored with careful attention to the QT interval in patients receiving intravenous pentamidine therapy, even though patients have no symptoms, and that an alternative regimen should be considered if the QT interval becomes prolonged or bradycardia appears. In addition, the electrolyte balance should be maintained with aggressive supplementation; patients who develop polymorphic ventricular tachycardia may benefit from magnesium sulfate injection and cardiac pacing.

\section{References}

1) Pearson RD, Hewlett EL. Pentamidine for the treatment of Pneumocystis carinii pneumonia and other protozoal diseases. Ann Intern Med 103: $782,1985$.

2) Mallory DL, Parillo JE, Bailey KR, et al. Cardiovascular effects and safety of intravenous and intramuscular pentamidine isethionate. Crit Care Med 15: 503, 1987.

3) Wharton JM, Demopulos PA, Goldschlager N. Torsade de pointes during administration of pentamidine isethionate. Am J Med 83: 571, 1987.

4) Gonzales A, Sager PT, Akil B, Rahimtoola SH, Bhandari AK. Pentamidine-induced torsade de pointes. Am Heart Journal 122: 1489, 1987.

5) Quadrel MA, Atkin SH, Jaker MA. Delayed cardiotoxicity during treat- 


\section{OTsuKA et al}

ment with intravenous pentamidine: two case reports and a review of the literature. Am Heart J 123: 1377, 1992.

6) Manı S, Kocheril AG, Andriole VT. Case report: pentamidine and polymorphic ventricular tachycardia revisited Am J Med Scı 305: 236, 1993.

7) Green PT, Reents S, Harman E, Curtıs AB. Pentamıdıne-1nduced torsades de pointes in a renal transplant recipient with Pneumocystls carınıl pneumonia. South Med J 83: 481, 1990.

8) Goa KL, Campol1-Rıchards DM. Pentamıdıne isethınate. A review of its antıprotozoal actıvity, pharmacokınetic properities and therapeutic use in Pneumocystls carinu pneumonia. Drug 33: 242, 1987.

9) Sands M, Kron MA, Brown RB. Pentamıdıne: a review. Rev Infect Dis 7: 625, 1985.

10) Steın KM, Haronian H, Mensah GA, Acosta A, Jacobs J, Klıgfield P. Ventricular tachycardia and torsades de pointes complicatıng pentamıdine therapy of Pneumocystls carını pneumonia in the acquired immunodeficiency syndrome Am J Cardıol 66: 888, 1990

11) Stein KM, Fenton $C$, Lehany AM, Okın PM, Kligfield $P$ Incidence of $Q T$ interval prolongation during pentamıdine therapy of Pneumocystıs carını pneumonia. Am J Cardıol 68: 1091, 1991

12) Cohen IS, Anderson DW, Vırmanı R, Reen BM, Macher AM, Sennesh J, Dilorenzo P, Redfield R Congestive cardiomyopathy in association with the acquired immunodeficiency syndrome NEngl J Med 315: 628, 1986.

13) Stratmann HG, Kennedy HL Torsades de pointes associated with drugs and toxins. recognition and management. Am Heart J 113: 1470, 1987.
14) Bibler MR, Chou TC, Toltzis RJ, Wade PA. Recurrent ventricular tachycardıa due to petamidine-induced cardıotoxicity. Chest 94: 1303, 1988.

15) Eisenhauer MD, Eliasson AH, Taylor AJ, Coyne PE Jr, Wortham DC. Incidence of cardiac arrhythmias durıng intravenous pentamidıne therapy in HIV-infected pat1ents. Chest 105: 389, 1994.

16) Krogstad DJ, Walzer PD, Western KA. Informational material for phys1crans. Pentamicine isethionate (Lomidine). United States Department of Health and Human Services. Atlanta, Georgia, p. 1-10, 1971.

17) Donnelly H, Bernard EM, Rothkotter H, Gold JW, Armstrong D. Distr1bution of pentamidine in patients with AIDS. J Infect Dis 157: 985, 1988.

18) Pujol M, Carratala J, Maurı J, Viladrich P. Ventrıcular tachycardıa due to pentamidine isethionate. Am J Med 84: 980, 1988 (letter).

19) Tzivonı D, Bana1 S, Schuger C, Benhorın J, Keren A, Gottlieb S, Stern S. Treatment of torsade de pointes with magnesium sulfate. Circulation 77: 392, 1987

20) Keren A, Tzivonı D, Golhman JM, Corcos P, Benhorin J, Stern S. Ventricular pacing in atypical ventricular tachycardıa. J Electrocardiol 14: 201, 1981

21) Ewy GA Electrical therapy for cardiovascular emergencies. Circulation 74: IV 111, 1986.

22) Taylor AJ, Hull RW, Coyne PE, Woosley RL, Elıasson AH. Pentamıdıneinduced torsades de pointes: safe completion of therapy with inhaled pentamıdıne Clın Pharmacol Ther 49: 698, 1991. 